REVISTA CIENCIAS BIOMÉ DICAS

ARTÍCULOS DE REVISIÓN

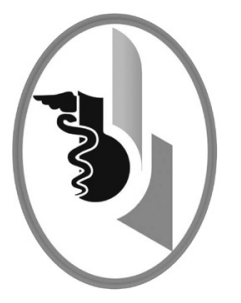

\title{
LA NEUROGLOBINA Y SU POTENCIAL RELACIÓN CON LA FUNCIÓN CEREBRAL Y EL SUEÑO*
}

\author{
THE POTENTIAL ROLE OF NEUROGLOBIN IN THE CEREBRAL \\ FUNCTION AND SLEEP
}

\author{
Acosta-Hernández Mario Eduardo ${ }^{1}$ \\ Rendón-Bautista Luis ${ }^{2}$ \\ Priego-Fernández Sergio ${ }^{3}$ \\ Peña-Escudero Carolina ${ }^{4}$ \\ Martínez-Cruz Betsy ${ }^{5}$ \\ Melgarejo-Gutiérrez Montserrat ${ }^{5}$ \\ García-García Fabio ${ }^{6}$
}

Correspondencia: fgarcia@uv.mx

Recibido para evaluación: febrero - 20 - 2015. Aceptado para publicación: agosto - $24-2016$

\section{RESUMEN}

Introducción: el sueño es un fenómeno biológico complejo en el que participan áreas del cerebro y neurotransmisores específicos. Recientemente se describió una proteína que pertenece a la familia de las globinas, llamada neuroglobina ( $\mathrm{Ngb}$ ), que se expresa en neuronas de los núcleos pedúnculo pontino tegmental y laterodorsal tegmental. Ambos núcleos son responsables de generar y mantener el sueño de movimientos oculares rápidos.

Objetivo: describir los principales hallazgos respecto al papel de la Ngb en la fisiología nerviosa y su potencial rol en el ciclo vigilia/sueño.

Materiales y métodos: revisión temática a conveniencia en artículos presentes en bases de datos virtuales, se consideraron estudios en diferentes modelos animales 0 piezas de laboratorio.

Resultados: varios estudios señalan la importancia que tiene la $\mathrm{Ngb}$ en la fisiología del sueño. Se ha señalado específicamente que la privación total de sueño por 24 horas en la rata reduce el número de neuronas inmuno positivas a $\mathrm{Ngb}$ en los núcleos ya señalados, sugiriendo que la expresión de $\mathrm{Ngb}$ es dependiente de la presencia del sueño. Si bien este mecanismo no es del todo claro, podría ser regulado a través de la vía orexinérgica, específicamente en neuronas que expresan el receptor ORX-A.

Conclusión: la $\mathrm{Ngb}$ tiene un papel en la fisiología del sueño y la vigilia. Entre otras observaciones se sugiere que el sueño es el promotor de la síntesis de la Ngb que será utilizada durante la vigilia. Más estudios son necesarios para precisar el papel de $\mathrm{Ngb}$ en la fisiología del sueño. Rev.cienc.biomed. 2016;7(2):285-295.

\footnotetext{
* Un producto del proyecto de investigación: El sueño como regulador de la neuroglobina en el cerebro de la rata (CONACYT-254264).

1 Doctorado en Ciencias. Técnico Académico. Departamento de Biomedicina. Docente Facultad de Bioanálisis. Universidad Veracruzana. México.

2 Estudiante de Pregrado. Facultad de Biología. Universidad Veracruzana. México.

3 Estudiante de Pregrado. Facultad de Psicología. Universidad Veracruzana. México.

4 Estudiante de Posgrado. Maestría en Ciencias de la Salud. Universidad Veracruzana. México.

5 Doctorado en Ciencias. Investigador Titular. Facultad de Medicina. Universidad Veracruzana. México.

6 Doctorado en Ciencias Investigador Titular. Departamento de Biomedicina, Universidad Veracruzana. México.
} 
PALABRAS CLAVE

Privación de sueño; Estrés oxidativo; Globinas, Orexina.

\section{SUMMARY}

Introduction: sleep is a complex biological phenomenon in which participate different areas of the brain and specific neurotransmitters. Recently it was described a protein that belongs to a family of globins named neuroglobina. This protein works on tegmental pontine peduncle nucleus and laterodorsal tegmental. Both nuclei are responsible for generating and keeping quick ocular movements during the sleep.

Objective: to describe the main findings related to the role of neuroglobin in nervous physiology and its role in the cycle insomnia - sleep.

Materials y methods: it was carried out a revision case report by convinience in online articles. Different studies in animals and laboratory pieces were considered.

Resultados: different studies show the importance of $\mathrm{Ngb}$ in sleep physiology. It has been specifically pointed out that total sleep deprivation for 24 hours in the rat reduces the number of $\mathrm{Ngb}$-positive neurons in the nuclei already indicated, suggesting that $\mathrm{Ngb}$ expression is dependent on the presence of sleep. Although this mechanism is not entirely clear, it could be regulated through the orexinergic route, specifically in neurons expressing the ORX-A receptor.

Conclusions: $\mathrm{Ngb}$ has a role in the sleep and wakefulness physiology. Among other observations it is suggested that sleep is the promoter of the $\mathrm{Ngb}$ synthesis that will be used during wakefulness. More studies are needed to clarify the role of Ngb in sleep physiology. Rev.cienc.biomed. 2016;7(2):285-295.

\section{KEYWORDS}

Sleep deprivation; Oxidative stress; Globin; Orexin.

\section{INTRODUCCIÓN}

El sueño es un fenómeno biológico sincronizado con el ciclo luz/oscuridad que se alterna con la fase de vigilia. El sueño puede ser identificado por parámetros conductuales y registro de variables electrofisiológicas, lo que permite clasificar al sueño en dos etapas: sueño de ondas lentas y sueño de movimientos oculares rápidos $(1,2)$.

Cada una de estas etapas es regulada por áreas específicas del cerebro así como por neurotransmisores. Además, en un organismo que duerme, ocurre la liberación de hormonas y péptidos tanto a nivel central como a nivel periférico. El flujo sanguíneo cerebral y la temperatura central cambian de manera súbita en cada etapa de sueño, generando ciclos de actividad/inactividad metabólica a lo largo de la noche (2).

Se ha descrito una proteína de la familia de las globinas que se expresa en neuronas, denominada neuroglobina ( $\mathrm{Ngb})$. La expresión de la $\mathrm{Ngb}$ en el cerebro de los mamíferos se circunscribe a la corteza cerebral, hipocam- po, tálamo, hipotálamo y cerebelo, regiones que se caracterizan por un metabolismo celular intenso y por mayor consumo de $\mathrm{O}_{2}$. Por lo tanto, una de las funciones de la $\mathrm{Ngb}$ es su participación como transportador de $\mathrm{O}_{2}$ en el interior de las neuronas $(1,2)$.

Además se ha observado que la $\mathrm{Ngb}$ se expresa especialmente en regiones del cerebro involucradas en el ciclo vigilia/ sueño, regulación circadiana y regulación del apetito. De manera particular se encontró alto número de células positivas a $\mathrm{Ngb}$ en los núcleos pedúnculo pontino tegmental (PPTg) y laterodorsal tegmental (LDTg), ambos responsables de generar y mantener el sueño de movimientos oculares rápidos $(1,3,4)$.

Las neuronas positivas para $\mathrm{Ngb}$ co-expresan receptores de orexinas, las cuales son péptidos que se sintetizan en el hipotálamo lateral $(\mathrm{HL})$ y son responsables de inducir y mantener la vigilia (1-4). El objetivo de la revisión fue describir las características y funciones de la $\mathrm{Ngb}$ en la fisiología del sistema nervioso y su potencial asociación con el ciclo vigilia/sueño. 
ISSN: 2215-7840, 7(2), julio-diciembre 2016, Acosta-Hernández Mario Eduardo, Rendón-Bautista Luis, Priego-Fernández Sergio,

Peña-Escudero Carolina, Martínez-Cruz Betsy, Melgarejo-Gutiérrez Montserrat, García-García Fabio

\section{MATERIALES Y MÉTODOS}

Revisión temática en artículos obtenidos a conveniencia en bases de datos virtuales. No se especificó el lapso de tiempo a considerar ni se realizó calificación de la calidad de las fuentes. Se incluyeron estudios animales en modelos celulares y en la teorización derivada.

\section{RESULTADOS}

Diferentes modelos o piezas de laboratorio se han propuesto para el estudio del sueño, los núcleos cerebrales involucrados, los diferentes neurotransmisores y las fases identificadas. Las ratas son modelos proferidos para dichas exploraciones (3).

\section{Aspectos generales del ciclo vigilia/} sueño: el sueño es un fenómeno generado de manera activa por el cerebro y regulado por factores circadianos y homeostáticos. Es un proceso fisiológico complejo, caracterizado por reducción de la actividad motora, disminución de la respuesta a los estímulos sensoriales y por una serie de posturas estereotipadas. El sueño se distingue de otros estados "alterados" de la conciencia como la anestesia o el estado de coma, ya que es un estado reversible y autoregulable (1).

Las etapas que componen al ciclo vigiliasueño presentan características fisiológicas y conductuales bien definidas. Los criterios actuales de clasificación del ciclo vigiliasueño en roedores se dividen en: vigilia, sueño de ondas lentas (SOL) y sueño de movimientos oculares rápidos (SMOR). La determinación de estas etapas se realiza mediante diferentes criterios. [A] El conductual, incluye la apertura o cierre de los párpados y la posición corporal. [B] Los electrográficos, que comprenden el registro de la actividad eléctrica cortical usando el electroencefalograma (EEG) y el tono muscular a través del electromiograma (EMG) (Figura No 1).

Durante la vigilia, la actividad cortical registrada es desincronizada con ondas de alta frecuencia entre $14-30 \mathrm{~Hz}$ y de baja amplitud de 30 a $50 \mathrm{mv}$, denominadas ondas beta. Durante la transición entre la vigilia

\section{FIGURA NO 1. \\ ESTEREOTIPO CONDUCTUAL Y ACTIVIDAD ELECTROGRÁFICA DE LAS ETAPAS DEL CICLO VIGILIA-SUEÑO DE LA RATA}

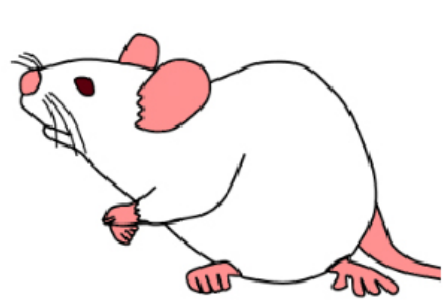

A) VIGILIA

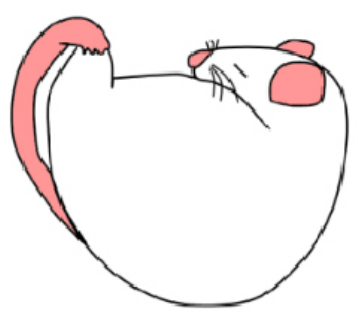

B) SOL

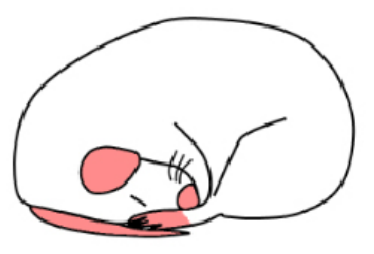

C) SMOR

EEG

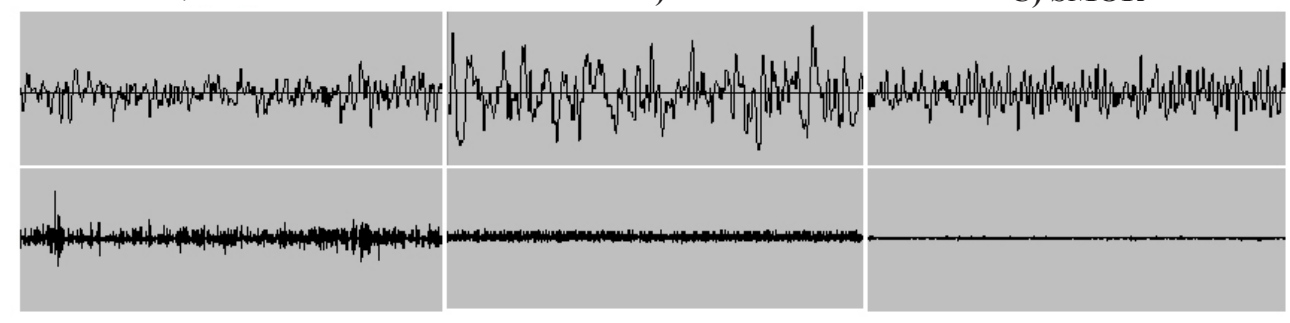

(A) Vigilia. Caracterizada por un estado conductual activo y respuesta a los estímulos sensoriales, además, presenta una actividad eléctrica cortical de ondas de alta frecuencia y baja amplitud, y un tono muscular elevado. (B) SOL. Se reduce el umbral sensorial y disminuye el tono muscular, el EEG muestra una actividad de ondas de baja frecuencia y alto voltaje.

(C) SMOR. Durante esta etapa se generan las ensoñaciones, aparece el ritmo theta hipocampal con ondas de alta frecuencia y baja amplitud, además de la desaparición del tono muscular. 
y el sueño, se presenta la etapa de vigilia quieta, caracterizada por una postura en reposo, reducción del tono muscular y cierre de los párpados. A nivel cortical el registro de EEG muestra ondas de bajo voltaje 100$200 \mu \mathrm{V}$ y una frecuencia entre los $8-12 \mathrm{~Hz}$, son las ondas alfa $(1,2,4,5)$.

En la fase SOL el registro del EEG muestra actividad con dominancia de ondas de baja frecuencia, $0.5-4.0 \mathrm{~Hz}$ y voltaje $<200 \mu \mathrm{V}$, se reduce el umbral sensorial y el tono muscular, aunque disminuye no desaparece. El sueño SMOR, también conocido como sueño paradójico, es caracterizado por actividad cortical similar a la vigilia con ondas de alta frecuencia: $4 \mathrm{a} 8 \mathrm{~Hz}$ y baja amplitud, denominadas ondas theta. El tono muscular desaparece por completo, sin embargo, ocurren descargas musculares repentinas llamadas mioclonías, las cuales aparecen en forma de espigas a lo largo del registro del EMG (2).

La organización cíclica del sueño varía entre especies, por ejemplo, en humanos las fases de sueño SMOR y SOL se alternan generando entre 4 y 5 ciclos de sueño a lo largo de una noche, cada ciclo tiene una duración aproximada de 90 minutos $(3,4)$. El ciclo de vigilia/sueño en roedores difiere de los humanos tanto en su organización como en su regulación circadiana, ya que estos son de hábitos nocturnos, es decir, la mayor parte del ciclo de luz se encuentra en reposo o sueño, y durante la noche son más activos. De igual manera, las ratas, así como otros mamíferos, son polifásicas, lo cual significa que presentan varios ciclos de sueño durante un período de 24 horas. En este sentido, el uso de roedores en el laboratorio los hace un modelo ideal para el estudio del sueño, ya que su ciclo puede ser controlado bajo condiciones específicas de luz/oscuridad (5).

Circuito anatómico cerebral de la vigilia y el sueño: el circuito anatómico y neuroquímico que regula el ciclo vigilia/sueño se localiza en el tallo cerebral, hipotálamo, tálamo y corteza. El núcleo supraquiasmático (NSQ) es un grupo de neuronas que se localiza en la base del hipotálamo al lado del tercer ventrículo y por arriba del quiasma óptico. EI NSQ es considerado el reloj interno de los mamíferos y es responsable de la presencia de los ritmos circadianos $(6,7)$. La lesión del NSQ suprime los ritmos circadianos, lo cual deriva en una desincronización de los procesos fisiológicos, incluyendo el ciclo vigilia/sueño (8).

Bajo condiciones normales, el NSQ se "reinicia" de manera diaria por estímulos luminosos provenientes de la retina durante el día y por la secreción de melatonina por parte de la glándula pineal durante la noche $(9,10)$. Las señales luminosas se reciben a través de un conjunto especializado de células ganglionares localizadas en la retina que contienen el fotopigmento melanopsina (11). Estas señales mantienen en sincronía el reloj endógeno con el ciclo luz/oscuridad.

La relación entre el NSQ y el sueño ha sido objeto de numerosas investigaciones. EI NSQ envía proyecciones hacía el núcleo preóptico ventrolateral (VLPO) y a las neuronas del hipotálamo lateral (HL) (12-15). EI VLPO está asociado al mantenimiento del SOL y el $\mathrm{HL}$ al mantenimiento del despertar. Cuando el factor luz se apaga el NSQ activa a las neuronas gabaérgicas del VLPO induciendo sueño. En contraste, cuando la luz se enciende, el NSQ activa a las neuronas orexinérgicas del $\mathrm{HL}$ favoreciendo el despertar. Sin embargo, el circuito es más complejo porque el NSQ también envía proyecciones hacia la zona adyacente subparaventricular (SPZ) que a su vez, envía un número reducido de proyecciones hacia las neuronas del núcleo VLPO y del HL (12-16).

Adicionalmente, la SPZ envía aferencias al núcleo dorso medial hipotalámico (DMH) (17-19). El núcleo DMH contiene neuronas de naturaleza glutamatérgica que envía proyecciones excitatorias a las neuronas orexinérgicas del $\mathrm{HL}$ para inducir el despertar (16,18-20). El núcleo DMH envía también proyecciones hacia las neuronas de los núcleos PPTg y LTDg, los cuales participan en la vigilia y en SMOR. De igual manera, las neuronas orexinérgicas del $\mathrm{HL}$ envían un número importante de proyecciones hacia los núcleos PPTg y LDTg inhibiendo su actividad durante el SMOR y en consecuencia, favoreciendo el despertar por medio de la activación de regiones como el área ventrotegmental (VTA) (20-22). 


\section{FIGURA No 2. CIRCUITO ANATÓMICO Y NEUROQUÍMICO DEL CICLO VIGILIA/SUEÑO}

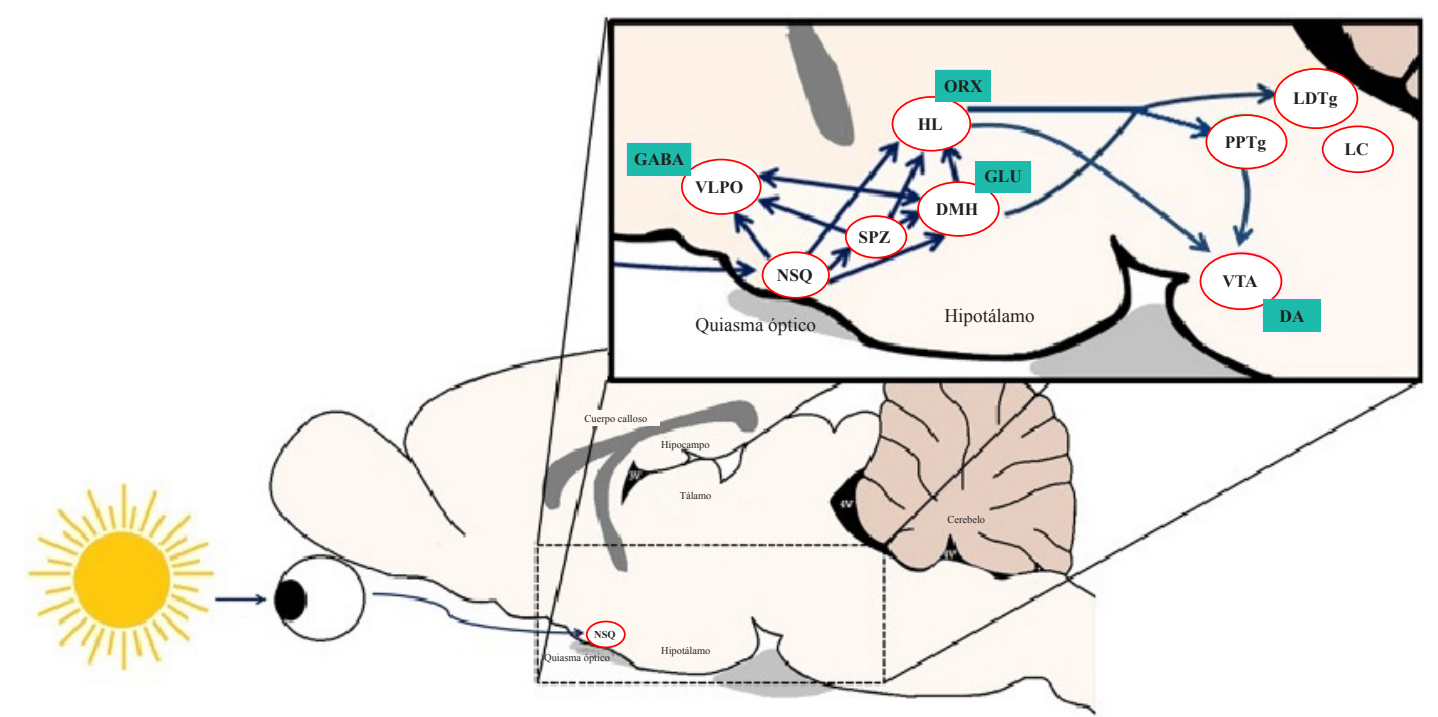

El estímulo luminoso es captado por la retina, donde pasa a las células ganglionares, luego la señal llega al NSQ que envía proyecciones hacia las siguientes áreas: a las neuronas gabaérgicas (GABA) del VLPO, las neuronas orexinérgicas (ORX) del HL, las neuronas glutamatérgicas (GLU) del DMH y hacia la SPZ. La SPZ envía proyecciones hacia el VLPO, el HL y el DMH, este último también manda proyecciones a dichos núcleos y, al igual que el HL, envía otras hacia núcleos del tallo cerebral como PPTg y LDTg. El núcleo PPTg y el HL también envían proyecciones a las neuronas dopaminérgicas (DA) del VTA.

La familia de las globinas: las globinas son una familia de metaloproteínas que se caracterizan por su forma globular. De manera general, están formadas por 8 unidades a $(\mathrm{A}-\mathrm{H})$ que muestran una disposición o plegamiento característico $(23,24)$. Todas las globinas contienen un grupo prostético (Fe-protoporfirina XI), mediante el cual forman uniones reversibles con ligandos gaseosos como $\mathrm{O}_{2}$, $\mathrm{CO}$ y NO. La mayoría de las globinas cumplen funciones respiratorias suministrando y almacenando oxígeno en las células. Filogenéticamente son moléculas antiguas y comparten características en común, los diferentes eventos evolutivos han generado una diversidad de globinas que están presentes en bacterias, hongos, plantas, animales vertebrados e invertebrados $(23,25)$.

Hasta el momento han sido identificados cinco tipos de globinas $(25,26)$. La hemoglobina $(\mathrm{Hb})$, localizada en los eritrocitos, tiene la función de transportar $\mathrm{O}_{2}$ desde los pulmones a los tejidos a través del sistema circulatorio y $\mathrm{CO}_{2}$ en sentido inverso. La mioglobina ( $\mathrm{Mb}$ ) se encuentra en el músculo estriado y cardíaco, donde actúa almacenando $\mathrm{O}_{2}$, facilitando la difusión intracelular del mismo hacia la mitocondria. La Mb posee una importante función como dioxigenasa al biotransformar los radicales de $\mathrm{NO}$ en nitritos $(23,25)$.

Por otra parte, la Ngb y la citoglobina (Cygb) son dos globinas descubiertas recientemente en vertebrados $(23,27)$. La $\mathrm{Ngb}$ se expresa principalmente en el tejido neuronal, teniendo como función principal la de suministrar $\mathrm{O}_{2}$ al tejido cerebral $(23,27)$. Experimentos realizados in vivo e in vitro han mostrado que la $\mathrm{Ngb}$ posee un papel neuroprotector al sobreexpresarse en situaciones de hipoxia, como puede ser en el infarto cerebrovascular (27). Para el caso de la Cygb se cree que su actividad biológica está relacionada con la síntesis de colágeno (28). Recientemente fue identificada en peces y anfibios un nuevo tipo de globina llamada globina X (GbX) (27-28).

Estructura de la Ngb: es una proteína monomérica constituida por 150 aminoácidos y un peso aproximado de $17 \mathrm{kDa}$, fue 
descubierta inicialmente en mamíferos como el ratón y en el ser humano, posteriormente, se encontró en rata, pez globo, así como en pez cebra, lo que sugiere una amplia distribución en todas las especies de vertebrados (29).

La Ngb comparte entre el 20 y el $25 \%$ de la secuencia de aminoácidos comparada con el resto de las globinas homólogas presentes en otros vertebrados. A pesar de estas diferencias, la $\mathrm{Ngb}$ adopta un plegamiento tridimensional idéntico al resto de las globinas ( 8 subunidades designadas de $A-H$ ) (Figura No 3).

La $\mathrm{Ngb}$ puede unirse al $\mathrm{O}_{2}$ de manera reversible $(23,29,30)$. La característica estructural más importante de esta proteína es el enlace hexacoordinado que forma el grupo hemo con el átomo de Fe en su estado férrico $\left(3^{+}\right)$y ferroso $\left(2^{+}\right)(25,29)$. Estudios cristalográficos confirman que al no existir ligandos externos, la histidina en la posición 7 de la hélice $E$ (HisE7) se une con el Fe del grupo hemo en esa posición, generando que cualquier ligando externo en estado gaseoso

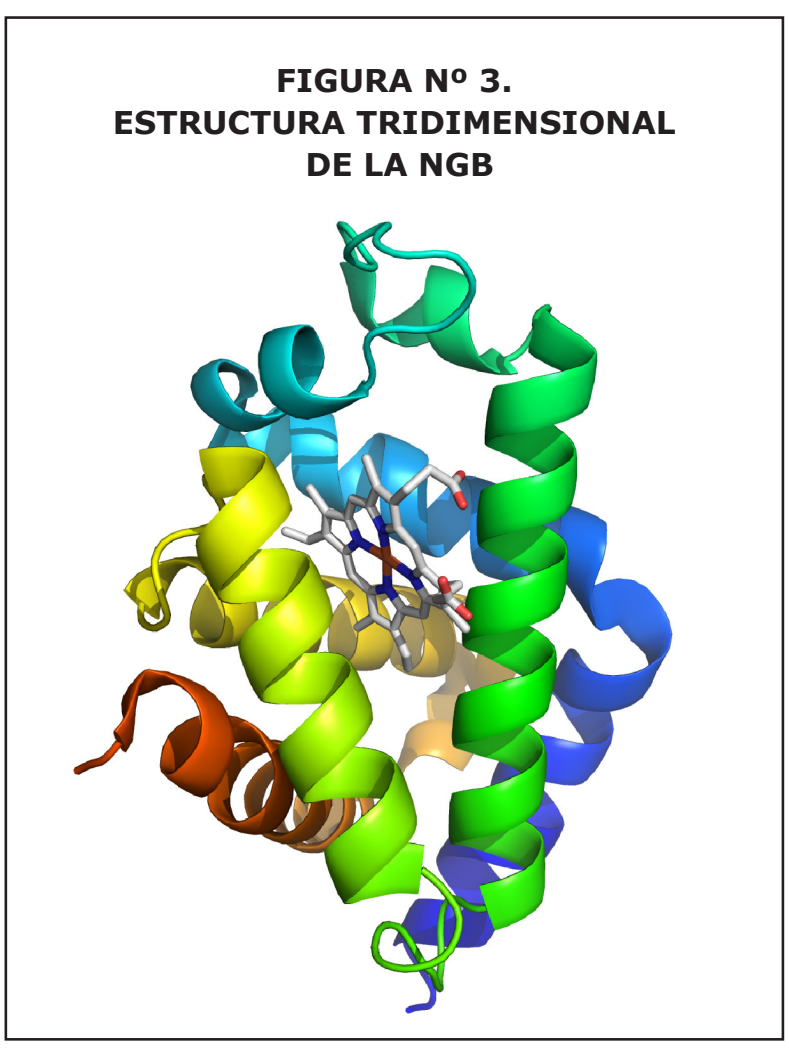

Tomado de Vallone et al. 2004 (31). como por ejemplo, $\mathrm{O}_{2}$ y $\mathrm{CO}_{2}$ compitan con el ligando interno (E7) para su unión con el átomo de $\mathrm{Fe}$ (23). Por otra parte, la hexacoordinación de la $\mathrm{Ngb}$, como la de otras globinas, hace que la unión al ligando sea independiente de variaciones en la temperatura; su estructura protege al átomo de $\mathrm{Fe}^{3+}$ de moléculas oxidantes evitando que se forme el radical ferrilo $\left(\mathrm{Fe}^{4+}\right)$, del que se sabe que es altamente citotóxico (23).

\section{Expresión de la $\mathbf{N g b}$ en el sistema} nervioso: la $\mathrm{Ngb}$ se expresa en concentraciones micromolares en el SNC y periférico, por lo que representa el $0.01 \%$ del contenido total de proteínas en el cerebro y en otros tejidos endócrinos $(29,30,32,33)$. Particularmente, en la retina, se encuentran altas concentraciones de esta proteína, lo cual puede deberse a que es uno de los tejidos del cuerpo que más consume $\mathrm{O}_{2}(29)$.

El grado de expresión de la $\mathrm{Ngb}$ en el cerebro humano varía (29), se expresa en áreas del cerebro como corteza cerebral, hipocampo, tálamo, hipotálamo y cerebelo $(33,34)$, regiones que se caracterizan por un metabolismo celular intenso y por mayor consumo de $\mathrm{O}_{2}$ (34). De manera particular, se encontró un alto número de células positivas de $\mathrm{Ngb}$ en el núcleo LDTg, PPTg, núcleo preóptico, amígdala, LC y el NSQ $(35,36)$.

Funciones de $\mathbf{N g b}$ : se le atribuyen varias. Suministro de $\mathrm{O}_{2}$ a las células de tejido nervioso y función enzimática comparable con NADH oxidasa que facilita la producción de ATP. Figura No 4. Así mismo, controla los niveles de $\mathrm{O}_{2}$ y participa en la vía de transducción de señales, uniéndose a las proteínas $\mathrm{G}$ e inhibiendo la disociación de los nucleótidos de guanina. Elimina sustancias tóxicas para las células como las especies de oxígeno reactivas (ROS) y nitrógeno, también la eliminación de NO (37-41). Debido a su expresión en áreas específicas del cerebro se cree que puede relacionarse en la regulación del ciclo vigilia/sueño $(35,36)$.

Función antioxidante del sueño y Ngb: el metabolismo neuronal requiere la oxidación de glucosa, así como concentraciones adecuadas de $\mathrm{O}_{2}$ que se ven incrementadas con la actividad celular. Durante el 


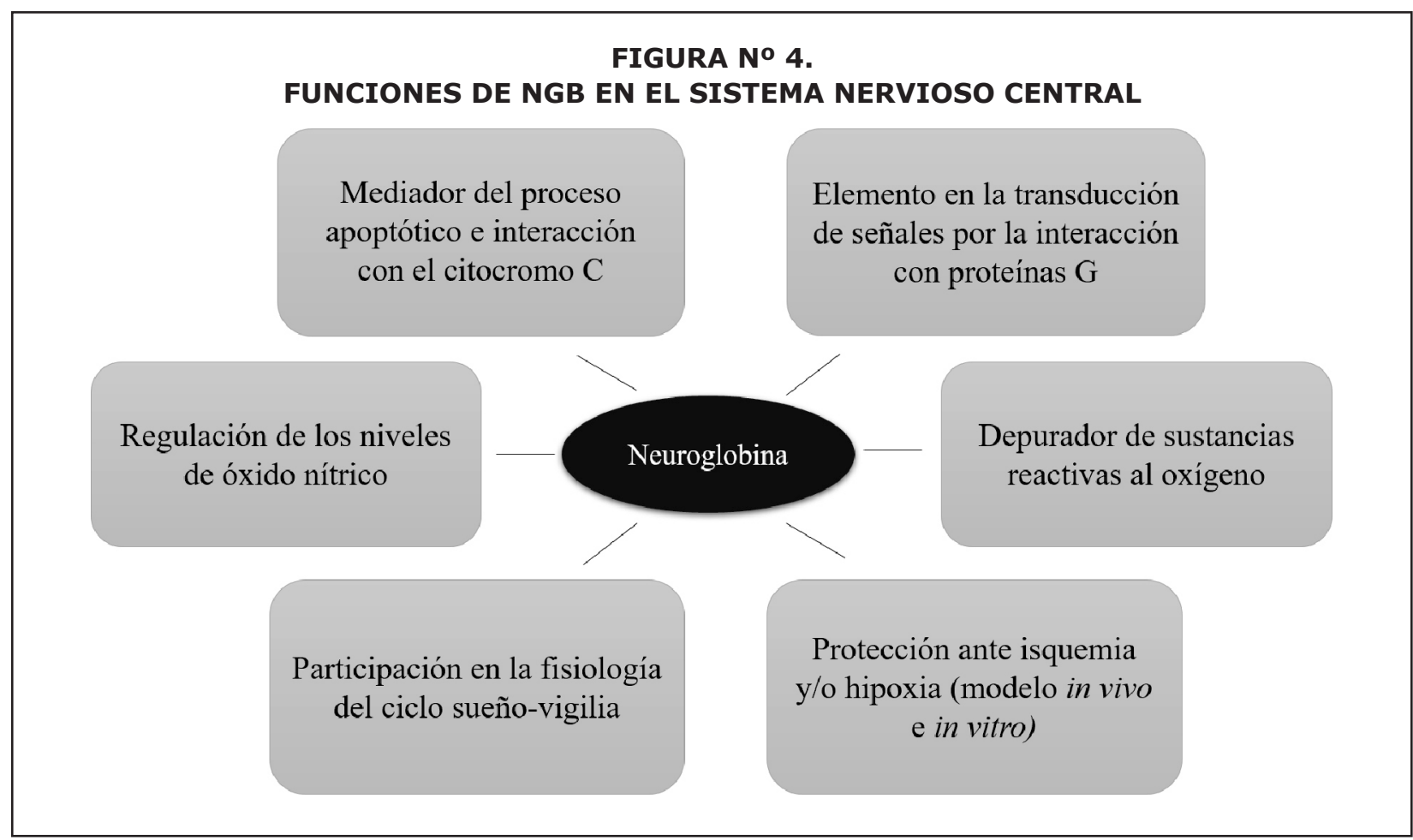

metabolismo celular se producen ROS como los aniones superóxido $\left(\mathrm{O}_{2}^{-}\right)$, radicales hidróxilo $\left(\mathrm{OH}^{-}\right)$, peróxido de hidrógeno $\left(\mathrm{H}_{2} \mathrm{O}_{2}\right)$, entre otros (42).

En el sistema nervioso, como en la mayoría de los tejidos, las concentraciones de ROS son reguladas por diversos mecanismos antioxidantes en donde participan macromoléculas como el glutatión y las enzimas glutatión peroxidasa y la superóxido dismutasa. Sin embargo, cuando las concentraciones de ROS superan la capacidad del sistema para mantener la homeostasis celular se produce un daño a nivel celular conocido como estrés oxidativo. A esta condición se le ha asociado con la apoptosis, debido a que ROS se unen de manera covalente con proteínas presentes tanto en membrana celular y en ácidos nucleicos, lo cual imposibilita que la célula repare los daños y desencadene la apoptosis, que finalmente ocasiona la muerte de la célula $(42,43)$.

Cuando aumenta la etapa de vigilia, el estrés oxidativo puede ocasionar daño en las neuronas de estructuras cerebrales como tálamo, hipotálamo e hipocampo al incrementarse las concentraciones de ROS y disminuir las concentraciones de glutatión reducido y la actividad de la superóxidodismutasa (42).

Una de las funciones del sueño es su acción antioxidante a nivel de sistema nervioso, lo que permite la restauración del tejido cerebral ocasionado por el metabolismo celular durante la etapa de vigilia. Esta función puede estar relacionada con la actividad de la $\mathrm{Ngb}$ al eliminar sustancias tóxicas para las células como los ROS y nitrógeno, y la eliminación de NO $(42,43)$.

La Ngb puede inhibir la enzima RacGTPasa, evitando la reorganización del citoesqueleto, lo cual es necesario para que se lleve a cabo la apoptosis (38). Proceso complejo en el que el citocromo $\mathrm{C}$ es liberado desde la mitocondria e interactúa con la proteína Apaf-1 [proteasa activada del factor 1 de apoptosis] en presencia de desoxiadenosinatrifosfato (dATP) para formar una compleja macromolécula llamada apoptosoma. Una vez formado el complejo, la célula activa la cascada de señalización intracelular modificando la permeabilidad de la membrana y finalmente, la activación de la caspasa 3 llevará a la muerte de la célula. Existe la hipótesis de que la Ngb puede modular la muerte celular al intervenir en 
la vía apoptótica al reducir la liberación del citocromo C. De igual manera, la Ngb puede inhibir RacGTPasa y Pak-1, previniendo el rearreglo del citoesqueleto necesario para que se lleve a cabo la apoptosis $(41,43)$. Por lo que la expresión de $\mathrm{Ngb}$ en las células pudiera estar directamente relacionada con la supervivencia celular.

Ngb y el sueño: como ya se mencionó, la $\mathrm{Ngb}$ se localiza predominantemente en neuronas y su distribución en el cerebro se circunscribe a regiones muy específicas $(44,45)$. Particularmente se expresa en áreas del cerebro involucradas en el control del ciclo vigila/sueño. El LC, el núcleo PPTg, el núcleo LDTg y el núcleo preóptico medial (MPO) expresan significativamente células positivas a $\mathrm{Ngb}$ (47). Las neuronas de dichos núcleos modifican su frecuencia de disparo durante el sueño o la vigilia (46). En un estudio realizado en el laboratorio se demostró que el número de células positivas a $\mathrm{Ngb}$ se reducen significativamente en los núcleos PPTg, LDTg y en el área periventricular (Pe) en animales privados de sueño por 24 horas, en comparación con el grupo no privado de dormir (36) (Tabla No 1).

Los niveles plasmáticos de corticosterona en los animales privados de sueño no se incrementaron en comparación con los animales no privados (36). Lo que sugiere que el estrés no es un factor condicionante para la expresión de la proteína, esto implica que la falta de sueño es el factor responsable de su reducción (36). En este sentido, en el mismo estudio se evaluó si la privación de sueño producía estrés oxidativo en el tejido cerebral, debido a que se ha reportado que la falta de sueño favorece el estrés oxidativo y porque a la $\mathrm{Ngb}$ se le atribuyen propiedades antioxidantes en las neuronas (49). Los resultados demostraron que la privación de sueño por 24 horas no induce estrés oxidativo, medido a través de la generación de malondialdehído, un metabolito producto de la peroxidación de lípidos de membrana (36). En conjunto, estos resultados fortalecen la hipótesis de que la expresión de Ngb depende de la presencia del sueño debido a que su inhibición es independiente del estrés oxidativo o del estrés fisiológico.

Un posible mecanismo de regulación de la expresión de $\mathrm{Ngb}$ podría ser a través de la activación de la vía orexinérgica, orexina-A (OX-A) y orexina-B (OX-B). Las neuronas positivas a $\mathrm{Ngb}$ en los núcleos del tallo cerebral (LC, PPTg, LDTg,) expresan el receptor de orexina-A (49); las orexinas participan en el mantenimiento de la vigilia. Por ende, mantener en vigilia forzada a una rata incrementa los niveles de OX-A circulantes y la unión a su respectivo receptor. La inhibición de $\mathrm{Ngb}$ observada en las ratas privadas de sueño puede ser resultado de la activación de los receptores a orexinas presentes en los núcleos que expresan $\mathrm{Ngb}$. No obstante, es algo que debe ser investigado.

Recientemente un estudio evaluó los niveles plasmáticos de $\mathrm{Ngb}$ en pacientes con diagnóstico de apnea obstructiva de sueño (AOS), encontrando que los niveles de $\mathrm{Ngb}$ se incrementan proporcionalmente de acuerdo al grado de severidad del AOS (50), lo cual apoya la función de transporte de oxígeno de la $\mathrm{Ngb}$ a nivel periférico en condiciones de hipoxia asociada con desorden de sueño. El hecho de que la $\mathrm{Ngb}$ sea sintetizada de manera normal en regiones cerebrales involucradas en la regulación del sueño (51) y que la falta de sueño reduzca su expresión

TABLA No 1.

NÚMERO DE CÉLULAS INMUNO-POSITIVAS A NGB EN RATAS PRIVADAS DE SUEÑO

\begin{tabular}{|c|c|c|c|}
\hline Área & No-PS & PS & Valor significancia \\
\hline LDTg & $84.7 \pm 6.712$ & $60.0 \pm 5.68$ & $\mathrm{p}=0.04$ \\
\hline PPTg & $66.81 \pm 4.95$ & $38.0 \pm 2.62$ & $\mathrm{p}=0.02$ \\
\hline Pe & $85.72 \pm 6.44$ & $55.42 \pm 5.15$ & $\mathrm{p}=0.002$ \\
\hline
\end{tabular}

LDTg (Núcleo laterodorsal tegmental). PPTg (núcleo pedúnculo pontino tegmental).

Pe (núcleo periventricular del hipotálamo).

Diferencia entre el grupo no privado (No-PS) y el grupo de animales privados de sueño (PS).

Los valores son expresados en media \pm desviación estándar. 
ISSN: 2215-7840, 7(2), julio-diciembre 2016, Acosta-Hernández Mario Eduardo, Rendón-Bautista Luis, Priego-Fernández Sergio,

Peña-Escudero Carolina, Martínez-Cruz Betsy, Melgarejo-Gutiérrez Montserrat, García-García Fabio

(52) sugieren que la síntesis de esta proteína podría estar ligada a la presencia de sueño y ser parte de las proteínas intracelulares que regulan dicho fenómeno. Del mismo modo, diversos estudios han demostrado la expresión de $\mathrm{Ngb}$ en células del NSQ, adicionalmente coexpresan el gen de la proteína homóloga del período circadiano (PER1) y responden a estímulos fóticos a través del tracto retinohipotalámico mediante inervaciones de neuronas que expresan el neuropéptido. Lo anterior sugiere que las células que expresan $\mathrm{Ngb}$ en el NSQ responden a estímulos fóticos y no fóticos mediante esta vía (53).

A lo anterior se añade que estudios a nivel molecular han determinado que el RNAm de $\mathrm{Ngb}$ muestra una variación diurna en el NSQ donde el máximo pico de expresión es el período de luz, pero se observa una disminución durante el período de oscuridad (54). Resultados preliminares del laboratorio demuestran que el mayor número de células positivas a $\mathrm{Ngb}$ se presentan durante el sueño y se reducen durante la vigilia, sugiriendo que el sueño es el promotor de la síntesis de la Ngb que será utilizada durante la vigilia.

En un estudio reciente realizado en ratones knockout para el gen de $\mathrm{Ngb}$ se encontró que someter a los ratones ante un estímulo lumínico durante la fase de oscuridad incrementa la expresión de PER1 en el NSQ así como la actividad locomotora (54). Estos resultados sugieren que la deficiencia de $\mathrm{Ngb}$ no afecta al reloj circádico, pero sí induce un efecto estimulante en la conducta asociado a un incremento de PER1.

\section{CONCLUSIÓN}

La Ngb es una hemoproteína monomérica de reciente descubrimiento en el cerebro de mamíferos y de otros vertebrados. Posee diversas funciones en SNC, se destaca su participación en el mecanismo de regulación homeostática de $\mathrm{O}_{2}$, NO y regulador del estrés oxidativo. Además de ser un potencial efector en la señalizacion intracelular asociada a procesos apoptóticos. Sumado a lo anterior, la $\mathrm{Ngb}$ tiene un papel en la fisiología del sueño y la vigilia, siendo el sueño importante promotor de la síntesis de la Ngb que será utilizada durante la vigilia. Si bien este mecanismo no es del todo claro, podría ser regulado a través de la vía orexinérgica, específicamente en neuronas que expresan el receptor ORX-A.

CONFLICTO DE INTERESES: ninguno que declarar.

FINANCIACIÓN: recursos propios de los autores.

\section{REFERENCIAS BIBLIOGRÁFICAS}

1. Fuller PM, Gooley JJ, Saper CB. Neurobiology of the sleep-wake cycle: sleep architecture, circadian regulation, and regulatory feedback. J Biol Rhythms. 2006;6:482-493.

2. Stenberg, D. Neuroanatomy and neurochemistry of sleep. Cell Mol Life Sc. 2007, 64:11871204.

3. Beersma DG. Models of human sleep regulation. Sleep Med Rev. 1998; 2:31-43.

4. Walker MP, Stickgold R. Sleep-dependent learning and memory consolidation. Neuron. 2004;44:121-133.

5. Timo-Iaria C, Negrão N, Schmidek WR, Hoshino K, Lobato de Menezes CE, Leme da Rocha T. Phases and states of sleep in the rat. PhysiolBehav. 1970; 5(9):1057-62.

6. Jin X, Shearman LP, Weaver DR, Zylka MJ, de Vries GJ, Reppert SM. A molecular mechanism regulating rhythmic output from the suprachiasmatic circadian clock. Cell. 1999;96:1-20.

7. Reppert SM. and Weaver DR. Coordination of circadian timing in mammals. Nature. 2002;418:935-941.

8. Moore RY. The suprachiasmatic nucleus and sleep-wake regulation. Postgrad Med. 2004;116(6 Suppl Primary):6-9.

9. Cassone VM, Chesworth MJ, Armstrong SM. Entrainment of rat circadian rhythms by daily injection of melatonin depends upon the hypothalamic suprachiasmatic nuclei. PhysiolBehav. 1986;36:1111-1121.

10. Johnson RF, Moore RY. and Morin LP. Loss of entrainment and anatomical plasticity after lesions of the hamster retinohypothalamic tract. Brain Res. 1988; 460:297-313.

11. Gooley J], Lu J, Fischer D, Saper CB. A broad role for melanopsin in nonvisual photoreception. J Neurosci. 2003;23:7093-7106. 
12. Watts AG, Swanson LW, Sanchez-Watts G. Efferent projections of the suprachiasmatic nucleus: I. Studies using anterograde transport of Phaseolus vulgaris leucoagglutinin in the rat. J Comp Neurol. 1987;258:204-229.

13. Chamberlin NL, Arrigoni E, Chou TC, Scammell TE, Greene RW, Saper CB. Effects of adenosine on GABAergic synaptic inputs to identified ventrolateral preoptic neurons. Neuroscience. 2003;119:913-918.

14. Sakurai T. Roles of orexin/hypocretin in regulation of sleep/wakefulness and energy homeostasis. Sleep Med Rev. 2005;4:231-241.

15. Yoshida K, McCormack S, España, RA, Crocker A, Scammell TE. Afferents to the orexin neurons of the rat brain. J Comp Neurol. 2006;5:845-861.

16. Lu J, Zhang YH, Chou TC, Gaus SE, Elmquist JK, Shiromani P, Saper, CB. Contrasting effects of ibotenate lesions of the paraventricular nucleus and subparaventricular zone on sleepwake cycle and temperature regulation. J Neurosci. 2001;21:4864-4874.

17. Deurveilher $\mathbf{S}$ and Semba K. Indirect projections from the suprachiasmatic nucleus to major arousal-promoting cell groups in rat: implications for the circadian control of behavioural state. Neuroscience. 2005;130:165-183.

18. Chou TC, et al. Critical role of dorsomedial hypothalamic nucleus in a wide range of behavioral circadian rhythms. J Neurosci. 2003;23:10691-10702.

19. Chou TC, Bjorkum, AA, Gaus SE, Lu J, Scammell TE, Saper, CB. Afferents to the ventrolateral preoptic nucleus. J Neurosci. 2002;22:977-990.

20. Thompson R, Swanson LW, Canteras N. Organization of projections from the dorsomedial nucleus of the hypothalamus: a PHA-L study in the rat. J Comp Neurol. 1997;376:143-173.

21. Peyron C, Tighe DK, Van den Pol AN, De Lecea L, Heller HC, Sutcliffe JG, Kilduff TS. Neurons containing hypocretin (orexin) project to multiple neuronal systems. J. Neurosci. 1998;18:9996-10015.

22. Chemelli RM, et al. Narcolepsy in orexin knockout mice: molecular genetics of sleep regulation. Cell. 1999;98:437-451.

23. Hankeln T, Ebner B, Fuchs C, Gerlach F, Haberkamp M, Laufs T, Roesner A, et. al. Neuroglobin and cytoglobin in search of their role in the vertebrate globin family. Journal of Inorganic Biochemistry. 2005;99:110-119.

24. Marchler-Bauer A, Derbyshire MK, Gonzales NR, Lu S, Chitsaz F, Geer LY, Geer RC, et. al. CDD: NCBI's conserved domain database. Nucleic acids res 2015 Jan;43 (Database issue):D222-6. doi: 10.1093/nar/gku1221. Epub 2014 Nov 20.

25. Kugelstadt D, Haberkamp M, Hankeln T, Burmester T. Neuroglobin, cytoglobin, and a novel, eye-specific globin from chicken Biochemical and Biophysical. Research Communications 2004;325:719-725.

26. Burmester T, Haberkamp M, Mitz S, Roesner A, Schmidt M, Ebner B, Gerlach F, et. al. Neuroglobin and cytoglobin: genes, proteins and evolution. Life, 2004; 56(11-12):703-707.

27. Brunori M and Vallone B. Neuroglobin, seven years after. Cell. Mol. Life Sci. 2007;64:1259-1268.

28. Roesner A, Fuchs C, Hankeln T and Burmester T. A globin gene of ancient evolutionary origin in lower vertebrates: Evidence for two distinct globin families in animals. Mol. Biol. Evol. 2005;22:12-20.

29. Pesce A, Dewilde S, Nardini M, Moens L, Ascenzi P, Hankeln T, Burmester T and Bolognesi. Human brain neuroglobin structure reveals a distinct mode of controlling oxygen affinity. Structure. 2003;11:1087-1095.

30. Lin YW, Wang J. Structure and function of heme proteins in non-native states: a mini-review. Journal of Inorganic Biochemistry. 2013;129:162-171.

31. Vallone B., Nienhaus K., Brunori M., Nienhaus G.U. The structure of murine neuroglobin: novel pathways for ligand migration and binding. Proteins. 2004; (56):85-92.

32. Wystub S, Laufs T, Schmidt M, Burmester T, Maas U, Saaler-Reinhardt S, Hankeln T, Reuss S. Localization of neuroglobin protein in the mouse brain. Neuroscience Letters. 2003;346:114-116.

33. Chen X, Liu Y, Zhang L, Zhu P, Zhu H, Yang Y, Guan P. Long-term neuroglobin expression of human astrocytes following brain trauma. Neuroscience Letters. 2015;606:194-199.

34. Pesce A, Dewilde S, Nardini M, Moens L, Ascenzi P, Hankelnd T, Burmestere T, Bolognesi $M$. The human brain hexacoordinatedneuroglobin three-dimensional structure. Micron. 2004;35:63-65.

35. Forrellat-Barrios M, Hernández-Ramírez P. Neuroglobina: nuevo miembro de la familia de las globinas. Revista Cubana de Hematología, Inmunología y Hemoterapia. 2011;27(3):291-296.

36. Melgarejo-Gutiérrez M, Acosta-Peña E, Venebra-Muñoz A, Escobar C, Santiago-García J and Garcia-Garcia F. Sleep deprivation reduces neuroglobin immunoreactivity in the rat brain. Neuroreport. 2013;24(3):120-125.

37. Hundahl CA, Allen GC, Nyengaard SD, Douglas Carter B, Kelsen J, Hay-Schmidt A. Neuroglobin in the rat brain: localization. Neuroendocrinology. 2008;88:173-182.

38. Dewilde S, Kiger L, Burmester T, Hankeln T, Baudin-Creuza V, Aerts T, Marden M, et al. Biochemical characterization and ligand binding properties of neuroglobin, a novel member of the globin family. J. biological chemistry. 2001; 42(19):38949-38955. 
ISSN: 2215-7840, 7(2), julio-diciembre 2016, Acosta-Hernández Mario Eduardo, Rendón-Bautista Luis, Priego-Fernández Sergio,

Peña-Escudero Carolina, Martínez-Cruz Betsy, Melgarejo-Gutiérrez Montserrat, García-García Fabio

39. Liu ZF, Zhang X, Qiao Y, Xu W, Ma C, Gu H, Zhou X, Shi L, Cui C, Xia D, Chen Y. Neuroglobin protects cardiomyocytes against apoptosis and cardiac hypertrophy induced by isoproterenol in rats. Int J Clin Exp Med. 2015;8(4):5351-5360.

40. Yu Z, Poppe JL and Wang X. Mitochondrial mechanisms of neuroglobin's neuroprotection. Oxid Med Cell Longev. 2013; 2013: 756989. doi: 10.1155/2013/756989.

41. Duong TT, Witting PK, Antao ST, Parry SN, Kennerson M, Lai B, Vogt S, Lay PA, Harris HH. Multiple protective activities of neuroglobin in cultured neuronal cells exposed to hypoxia reoxygenation injury. J Neurochem. 2009;108(5):1143-1154.

42. Hankeln T, Wystub S, Laufs T, Schmidt M, Gerlach F, Saaler-Reinhardt S, Reuss S, Burmester T. The cellular and subcellular localization of neuroglobin and cytoglobin - a clue to their function? IUBMB Life. 2004;56(11-12):671-679.

43. Acosta-Peña E, García-García F. Restauración cerebral: una función del sueño. Revista Mexicana de Neurociencia. 2009;10(4):274-280.

44. Fiocchetti M, De Marinis E, Ascenzi P, Marino M. Neuroglobin and neuronal cellsurvival. Biochimica et Biophysica Acta. 2013;1834:1744-1749.

45. Hundahl CA, Allen GC, Hannibal J, Kjaer K, Rehfeld JF, Dewilde S, et al. Anatomical characterization of cytoglobin and neuroglobin mRNA and protein expression in the mouse brain. Brain Res. 2010;1331:58-73.

46. Hundahl CA, Allen GC, Nyengaard JR, Dewilde S, Carter BD, Kelsen J, et al. Neuroglobin in the rat brain: localization. Neuroendocrinology 2008;88:173-182.

47. Szymusiak R, Alam N, McGinty D. Discharge patterns of neurons in cholinergic regions of the basal forebrain during waking and sleep. Behav Brain Res. 2000;115:171-182.

48. Gopalakrishnan A, Ji LL, Cirelli C. Sleep deprivation and cellular responses to oxidative stress. Sleep. 2004;27:27-35.

49. Everson CA, Henchen CJ, Szabo A, Hogg N. Cell injury and repair resulting from sleep loss and sleep recovery in laboratory rats. Sleep. 2014;37:1929-1240.

50. Xu M, Yang Y, Zhang J. Levels of neuroglobin in serum and neurocognitive impairments in Chinese patients with obstructive sleep apnea. Sleep Breath. 2012; Published on line 7 June, DOI 10.1007/s11325-012-0723-1.

51. Garry DJ, Mammen PP. Neuroprotection and the role of neuroglobin. Lancet. 2003;362:342-343.

52. Hundahl CA, Kelsen J, Dewilde S, Hay-Schmidt A. Neuroglobin in the rat brain (II): Colocalisation with neurotransmitters. Neuroendocrinology. 2008;88(3):183-198.

53. Hundahl CA, Hannibal J, Fahrenkrug J,Dewilde S,Hay-Schmidt A. Neuroglobin expression in the rat suprachiasmatic nucleus: colocalization, innervation, and response to light. J Comp Neurol. 2010;518(9):1556-69.

54. Hundahl CA, Fahrenkrug J, Hay-Schmidt A, Georg B, Faltoft B, Hannibal J. Circadian behaviour in neuroglobin deficient mice. PLoS One. 2012;7(4):e34462.

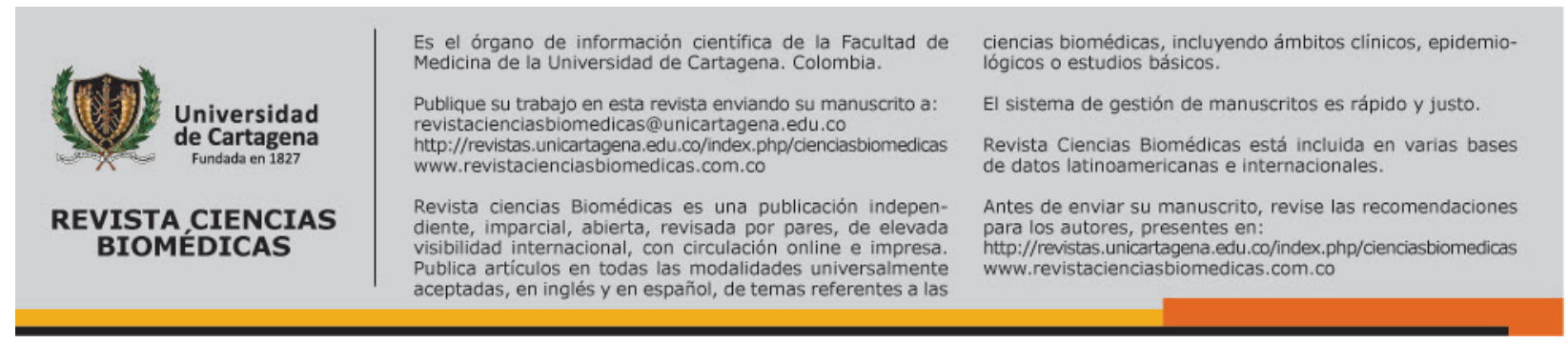

